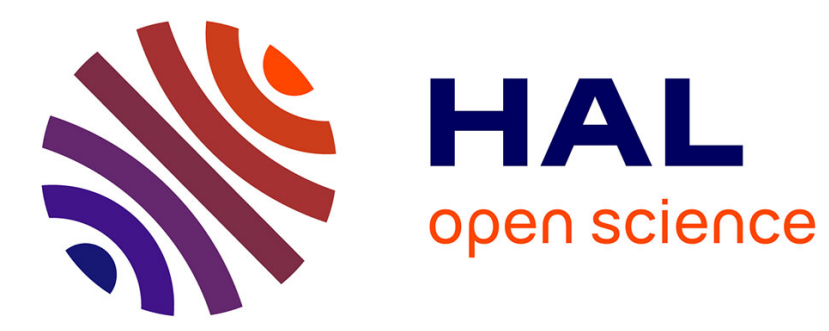

\title{
Explorer la rétraction dans l'espace: les services de soins dans le système urbain français
}

\author{
Sophie Baudet-Michel
}

\section{To cite this version:}

Sophie Baudet-Michel. Explorer la rétraction dans l'espace: les services de soins dans le système urbain français . Espace Géographique, 2015, 44 (4), 10.3917/eg.444.0369 . halshs-01436968

\section{HAL Id: halshs-01436968 \\ https://shs.hal.science/halshs-01436968}

Submitted on 6 Jun 2018

HAL is a multi-disciplinary open access archive for the deposit and dissemination of scientific research documents, whether they are published or not. The documents may come from teaching and research institutions in France or abroad, or from public or private research centers.
L'archive ouverte pluridisciplinaire HAL, est destinée au dépôt et à la diffusion de documents scientifiques de niveau recherche, publiés ou non, émanant des établissements d'enseignement et de recherche français ou étrangers, des laboratoires publics ou privés. 
Titre : Explorer la rétraction dans l'espace : les services de soins dans le système urbain français

Title : Exploring shrinking processes in space : health services in the French urban system

Baudet-Michel Sophie, «Explorer la rétraction dans l'espace : les services de soins dans le système urbain français », L'Espace géographique, 2015/4 (Tome 44), p. 369-380. DOI :

10.3917/eg.444.0369. URL : https://www.cairn.info/revue-espace-geographique-2015-4-page369.htm

UMR Géographie-cités, 13 rue du Four, 75006 Paris

sophie.baudet-michel@univ-paris-diderot.fr

Résumé : Cette position de recherche propose de transposer les cadres de la théorie spatiale de la diffusion des innovations à des phénomènes de rétraction en s'appuyant sur l'exemple de l'évolution de la répartition des services de soins dans le système urbain français. Les notions de concentration et de rétraction seront mobilisées pour explorer de manière systématique la dynamique spatiale des activités de soins. L'hypothèse d'une boucle de rétroaction positive entre dynamique spatiale des soins et dynamiques démographiques des villes est défendue.

Abstract : The following research position offers to transpose the spatial diffusion theory to shrinking processes. Processes of reconcentration and shrinking help to describe the location dynamic of medical services in the French urban system. The hypothesis to be tested is one of an systemic relation between demographic dynamics and medical services relocation.

Mots-clefs : diffusion, services de soins, système urbain, rétraction, concentration, processus, décroissance urbaine

Key words : diffusion, medical services, urban system, shrinking, polarisation, process, urban decline

Entre 1960 et 2014 l'effectif de la catégorie "Santé » du secteur des services en France métropolitaine est passé de 359000 à 1,2 millions d'emplois (une variation annuelle de 4,45\%). Au sein de cet ensemble, le nombre des médecins (libéraux et salariés) a augmenté de 60000 (1968) à 210000 (1990) et 219834 en 2014. Le nombre de médecins par habitant a ainsi été multiplié par 3, de 118,79 pour 100000 en 1968 à 334 en 2014'. En 2007, les généralistes sont présents dans $27 \%$ des communes, et accessibles à $95 \%$ de la population en

\footnotetext{
${ }^{1}$ En 1968 : environ 28000 généralistes libéraux et 18000 spécialistes libéraux ; en 2014 environ 62000 généralistes libéraux et 46000 spécialistes libéraux (répertoire ADELI). Source : Irdes, 7 août 2015 : http://www.irdes.fr/EspaceEnseignement/ChiffresGraphiques/Cadrage/DemographieProfSante/DemoMedecins.h tm
} 
moins de $15 \mathrm{mn}$. Ces communes sont essentiellement des pôles urbains et ruraux ${ }^{2}$ (Coldefy et al., 2011) et $85 \%$ des effectifs de ce secteur sont localisés dans les aires urbaines en 1990 (Paulus, 2005).

Paradoxalement cet accroissement de la place du secteur de la santé dans les activités économiques s'est accompagné depuis les années 1980 d'une réduction de la présence des soins sur le territoire: les transformations du système de soins impulsées par la puissance publique ont réduit le nombre de lits d'hospitalisation (de 604031 lits en 1981 à 508075 en 1999, Lucas-Gabrielli et Tonnelier, 2000), le nombre d'étudiants autorisés à poursuivre annuellement des études de médecine (de 9327 en 1978 à 3900 en 1999), le nombre d'établissements de santé. Les fermetures de maternité en constituent un exemple emblématique. En 2015 encore des fermetures étaient envisagées à Dourdan, Falaise, Millau.

C'est à cette réduction de la présence des soins dans l'espace des villes que je propose de m'intéresser i) en interrogeant l'évolution récente de la répartition spatiale des soins en m'appuyant sur les termes de rétraction et concentration, ii) en questionnant l'existence d'une relation entre dynamique des services de soins et dynamique démographique des villes, iii) en envisageant la réduction de la présence des soins comme un processus de diffusion spatiale.

\section{L'évolution récente de la présence des soins dans l'espace français : rétraction et concentration}

Avant de présenter les concepts de rétraction et de concentration et de développer les hypothèses qui sous-tendent ma position de recherche je commencerai par introduire le propos en décrivant l'évolution récente de la présence des soins dans l'espace français. La réduction de la présence des soins dans l'espace français constitue en effet le socle sur lequel mes hypothèses se sont construites.

De 1940 à 1970 l'Etat a organisé l'accès de tous à des structures de soins modernisées, en jouant à la fois sur le contrôle des formations et des structures hospitalières. Cette action volontariste s'est accompagnée d'une diffusion spontanée des professions médicales sur le territoire : le nombre de communes équipées en médecin est passé de 6637 (17\% des communes) en 1957 à 9166 en 1982 (25\% des communes) (Tonnelier, 1992) $)^{3}$. Fleuret et Séchet (2002) considèrent que les services de santé se sont propagés en deux phases : une diffusion des hôpitaux dans les années d'après-guerre, puis des médecins généralistes dans les années 1970-1980. La diffusion a été favorisée par la politique de santé très volontariste de l'Etat, par le contexte de croissance économique et les changements dans les modes de vie des ménages. Vigneron (2001) écrit qu'en 50 ans la croissance du nombre de médecins a profité à tous les départements même si les inégalités interdépartementales initiales se sont maintenues.

\footnotetext{
${ }^{2}$ Un pôle urbain est une unité urbaine offrant 5000 emplois ou plus et n'appartenant pas à la couronne périurbaine d'un autre pôle urbain. Un pôle rural compte 1500 emplois ou plus, polarise la population des communes voisines pour l'emploi, est situé dans l'espace à dominante rurale.

${ }^{3}$ Avant 1957, et en dépit d'une augmentation du nombre des médecins, le nombre de communes équipées évolue peu (6302 en 1900).
} 
Depuis les années 1980, et en dépit de l'augmentation du nombre des emplois rattachés au secteur de la santé, cette tendance à la diffusion semble s'être inversée et on assiste plutôt à une réduction de la présence des soins dans l'espace.

\section{Réduction du nombre d'établissements hospitaliers}

Engagée par la carte hospitalière (loi de 1970), la réduction de la présence hospitalière dans l'espace français a été ensuite largement mise en oeuvre avec les documents de programmation (Schémas Régionaux d'Organisation des Soins 1991-1999; 1999-2004; 2006-2011). Le nombre total d'établissements de santé est ainsi passé de 3650 établissements ${ }^{4}$ en 1975 (Labasse, 1980) à $2660^{5}$ en 2015. Les SROS ont constitué pour l'Etat un instrument de restructuration hospitalière qui a débouché sur la réduction du nombre de lits (loi Durieux 31.7.1991), la fermeture de nombreux services d'urgence, de maternité, et de chirurgie (IRDES, 2015). Entre 1995 et 2005, Vigneron et Haas (2009) ont compté 199 fermetures d'établissements de soins. En 2006, le rapport Vallancien (Delas, 2011) recommandait la fermeture de 113 services de chirurgie viscérale et orthopédique ${ }^{6}$. En 2010, un projet de décret ministériel prévoyait la fermeture de 54 services de chirurgie dans des établissements avec moins de 1500 actes annuels ${ }^{7}$. La réduction du nombre d'établissements de soins a pris différentes formes: suppressions de services ou fermetures d'établissements, transferts d'activité, reconversions, fusions juridiques (Lerouvillois et Vinclet, 2002) ${ }^{8}$. A titre d'exemple, le nombre de maternités est passé de 1369 en 1975 à 1010 en 1985, 814 en 1996, 554 en 2008 ; les maternités de moins de 200 lits et pratiquant moins de 300 accouchements par an ont été fermées (Lalande et al., 2012).

La réduction de l'offre de soins sur le territoire s'est aussi traduite par une transformation : baisse de la capacité d'accueil en hospitalisation complète (-15\% entre 1980-1996), réduction de la durée des séjours (moins de 6 jours en 2001 contre 20 en 1950), réduction du nombre de lits (10000 lits soit $1 / 3$ de la capacité hospitalière entre 1980 et 1996) au profit de l'hospitalisation de jour. Les établissements privés ont été les premiers à s'engager dans ces transformations (Lucas-Gabrielli et Tonnelier, 2000).

A l'échelle régionale, Lucas-Gabrielli et Tonnelier (2000) ont noté que les diminutions ont concerné toutes les régions, et qu'elles ont été plus importantes dans les régions les plus équipées, conduisant à une réduction des inégalités inter-régionales pour la chirurgie et la médecine : en PACA la réduction (fermeture des lits 1987-1997) a été de 24,07\%, en Picardie de $14 \%$, en Bretagne de 11\%. Dans une région bien pourvue en services de soins, les Pays-de-

\footnotetext{
${ }^{4}$ « La France disposait à la fin de 1973 de plus de 627000 lits répartis dans 3650 établissements.

L'hospitalisation privée y représentait alors 29\% des premiers et 75\% des seconds » (Labasse, 1980, p. 9). Labasse compte en 1973892 établissements publics pour 426400 lits dont la moitié est réservée au moyen et long séjour, et 2721 privés (Labasse, 1980, pp. 9-10).

${ }^{5}$ Source : http://www.drees.sante.gouv.fr/le-panorama-des-etablissements-de-sante-edition-2014,11413.html

${ }^{6}$ En raison de leur faible activité, et des pertes de chances pour les patients

${ }^{7}$ La fronde des élus et des directeurs d'établissements a conduit à reporter les fermetures d'abord envisagées.

${ }^{8}$ Entre 1977 et 1993111 établissements (30\%) des établissements ont participé à la recomposition dans un Bassin parisien élargi. Le sud a été moins touché (Lerouvillois et Vinclet, 2002).
} 
la-Loire, Jousseaume (2002) note qu'entre le début et la fin des années 1970, les services de chirurgie ou d'obstétrique avaient disparu de la plupart des bourgs de 4000 habitants. Pour l'auteur la réforme hospitalière des années 1990 a prolongé cette tendance, favorisée par l'augmentation de l'accessibilité aux grands centres urbains. Ainsi à la fin des années 1990 l'offre de chirurgie et d'obstétrique s'est trouvée concentrée dans une quinzaine d'agglomérations (environ trois par département). En 2000, les services hospitaliers n'étaient plus présents que dans les préfectures et sous-préfectures (Jousseaume, 2002). Fleuret (2002) signale d'ailleurs que cette logique rappelle la théorie des lieux centraux qui stipule que les hiérarchies urbaines sont régies par la portée des biens et services en fonction de seuils de population conférant ou non la rentabilité nécessaire au maintien des services. Les établissements hospitaliers et la médecine de spécialité obéissent en effet à cette logique hiérarchique (Barbat-Bussière, 2009 ; Tonnellier et Vigneron, 1999).

Dans ce contexte de réduction du nombre d'établissements, les densités de lits sont restées plus élevées dans les métropoles régionales, les disparitions ont concerné les petits établissements et les petites villes.

\section{Réduction de la présence de la médecine libérale dans l'espace}

A partir du début des années 1990, le départ à la retraite des nombreux médecins formés après-guerre, combiné à un numerus clausus bas pendant la période 1990-20009 a entraîné une stagnation des effectifs de médecins, un recul de leur présence dans les espaces ruraux, voire dans certaines zones urbaines. Indexé sur la capacité d'accueil des étudiants en stage hospitalier en $5^{\mathrm{e}}$ année, le numerus clausus a été fixé en 1971 à 8591 étudiants autorisés à poursuivre (entrée en $2^{\mathrm{e}}$ année) les études de médecine ${ }^{10}$. Il a baissé annuellement jusqu'en 1992 (3500) pour se stabiliser jusqu'en 2000 et remonter peu à peu jusqu'à 7500 à l'heure actuelle (http://www.irdes.fr/, Cadrage démographique, Septembre 2015). En dépit de l'élévation régionalisée du numerus clausus depuis 2000, l'observatoire National de la Démographie Médicale anticipe une baisse des professions de santé qui devrait durer jusqu'en 2020.

Coldefy et al. (2011) ont observé une phase de stagnation voire de diminution $(-0,8 \%)$ du nombre de communes équipées de soins de proximité à l'échelle de la France entière entre 1990 et 2006 alors qu'une forte diffusion $(+10,2 \%$ de communes équipées) a affecté l'ensemble de l'espace français entre 1980 et 1990 (Schmitt et Niel, 1999). Pour certaines spécialités (pédiatrie, ophtalmologie, psychiatrie, otorhinolaryngologie, chirurgie-dentaire, gynécologie) les distances moyennes d'accès ont légèrement augmenté entre 1990 et 2006 (Coldefy et al. 2011).

\footnotetext{
${ }^{9} 4000$ étudiants autorisés annuellement à poursuivre des études de médecine entre 1990 et 2000 . Afin d'amortir la diminution du nombre de médecins, le numerus clausus a été progressivement augmenté pour atteindre 8000 étudiants autorisés à poursuivre des études de médecine en 2008.

${ }^{10}$ Dans les années 1960, le nombre d'étudiants s'inscrivant en médecine augmente, et entre 1971 et 1977 environ 9000 étudiants poursuivent annuellement des études de médecine. La protestation des étudiants, et de certains syndicats, notamment le SNESUP diffère la baisse qui ne commencera réellement qu'en 1978.
} 
Jousseaume (2002) décrit en Pays-de-la-Loire à partir de 1990 un phénomène de concentration que signale une croissance plus rapide de l'offre médicale dans les villes moyennes et grandes au détriment des petites villes. Pour Alouis (2013) la spatialisation des médecins libéraux répond à des logiques commerciales et la recherche de la centralité est le déterminant principal du choix de localisation.

Un premier axe de ma recherche consistera à mettre en place les moyens d'observer et de formaliser l'évolution de la présence des services de soins afin de tester l'hypothèse d'une coexistence de phénomènes de concentration et de rétraction spatiale à l'échelon de l'ensemble du système urbain français, et à l'échelon local.

L'évolution sera envisagée sur les 4 décennies de la période 1982-2014 qui voient la mise en œuvre de la carte sanitaire, des documents de planification et de la tarification à l'acte. Les bases de données SNIIRAM de la CNAMTS et ADELI de la DRESS ${ }^{11}$ recensent la présence des praticiens depuis 1982, au niveau communal. Le fichier FINESS ${ }^{12}$ permet de suivre les établissements de soins. A partir de 1994, cette information peut être précisée (nombre de lits, nombre d'hospitalisation, nombre de lits par services ...) pour les établissements de santé grâce au fichier de Statistique Annuelle des Etablissements ${ }^{13}$. Une base de données harmonisée et détaillée de la présence de différentes catégories de soins sera élaborée.

Je postule que le système de soins, dont la place dans les activités françaises s'est accrue depuis les années 1970 (Paulus, 2004), se concentre dans l'espace, accroissant les inégalités $d$ 'accès aux soins entre les villes petites et moyennes ou grandes. Le terme de concentration en géographie est utilisé pour décrire des changements différentiels dans l'espace : lorsque les positions extrêmes (en valeur absolue) des espaces observés se renforcent et que ces espaces s'éloignent d'une position moyenne, on assiste à un phénomène de concentration spatiale (Pumain, Saint-Julien, 2001). Je m'intéresserai donc aussi à ces évolutions différentielles et

\footnotetext{
${ }^{11}$ SNIIRAM : Système National d'Information Inter Régime de l'Assurance Maladie et ADELI Automatisation Des Listes recense les professionnels relevant du code de la santé publique, du code de l'action sociale et des familles et des personnes autorisées à faire usage du titre de psychologue, d'ostéopathe, de psychothérapeute ou de chiropracteur. Caisse Nationale d'Assurance Maladie des Travailleurs Salariés. Le SNIIRAM est peu accessible et nous privilégierons donc la base ADELI.

${ }^{12}$ FINESS (Fichier National des Etablissements Sanitaires et Sociaux) a été mis en place en 1979. Géré par le ministère de la santé, il recense tous les établissements de santé ou sociaux (Centre de Lutte contre le Cancer, Urgences, Banque d'organes, Centres d'examen de santé, Maisons d'enfants ; PMI ; Handicap ; Médico-psy ; Foyers d'hébergement ; Maisons de retraites). Il est actualisé annuellement. Les données sont numériques à partir de 1984. Lorsque les établissements ferment ils disparaissent de la base. Les disciplines d'Equipement et de Prestation sont très détaillée jusqu'en 2000 et connue de manière plus agrégée après. Le nombre de lits ouverts est connu par discipline. Les établissements hospitaliers peuvent être clairement différenciés des autres car ils doivent nécessairement disposer de lits. L'année d'ouverture de l'établissement (géographique et juridique) est connue, avec une surreprésentation en 1980 (date attribuée lorsque la date d'ouverture de l'établissement était inconnue).

${ }^{13}$ L'enquête SAE est gérée par la DREES. Les établissements de santé recensés dans SAE étaient auparavant recensés dans le fichier Etablissements Hospitaliers Publics (EHP) et Etablissements Hospitaliers privés (EH80). recense près de 3000 établissements publics et privés. Ce fichier compte un millier d'établissements publics en 2006 (dont 31 Centres Hospitaliers Régionaux, 520 centres hospitaliers, 364 hôpitaux locaux (activité médicale seulement), 1067 établissements privés lucratifs en 2006 et 804 établissements privés non lucratifs (dont les établissements hospitaliers mutualistes, les centres de lutte contre le cancer). Le nombre de lits en Médecine, Chirurgie, Obstétrique est connu.
} 
j'essayerai de mesurer l'évolution du niveau de la concentration des services de soins dans le système urbain. J'utiliserai à la fois des indicateurs globaux tels que les coefficients de variation ou de dissymétrie, des coefficients de concentration (Gini, Theil) ${ }^{14}$, des indicateurs de primatie à différents niveaux (national, régional), de densité du semis des villes équipées en soins, mais aussi des indicateurs d'évolutions différentielle des équipements en soins des villes selon leur taille.

Le terme de rétraction est utilisé dans les travaux sur les «shrinking cities» ou «Schrumpfende Städte " qui appréhendent les processus de décroissance démographique et économique de villes allemandes, japonaises, américaines. Hélène Roth (2016) précise que l'expression «Schrumpfende Städte » émerge en Allemagne dès 1988 dans le contexte très spécifique des villes et régions est-allemandes en décroissance démographique. Le terme de «Schrumpfende », traduit en anglais par le terme "shrinking » et en français par celui de rétraction, fait alors référence à une perte de contenu, à une diminution de surface et de volume, au flétrissement, à l'altération du contenant. Il a une signification très descriptive et ne renvoie à aucun contexte théorique particulier. L'expression côtoie dans les travaux américains et français celles de «urban decline », de "déclin urbain », de "décroissance urbaine » voire de décroissance qui elles renvoient à des conceptions plus politiques du développement insistant sur l'idée que la croissance (démographique et économique) menace l'environnement physique et à terme l'humanité. En mobilisant ce terme à l'échelle interurbaine je souhaite caractériser l'évolution du semis spatial des services de soins et décrire la géographie des fermetures d'établissements publics et privés, de services, de lits, ou de cabinets de médecine libérale. L'utilisation de ce terme n'est pas indifférente car une de mes hypothèses est que la rétraction spatiale affecte davantage les villes en décroissance. Je développerai cette hypothèse dans une deuxième partie.

Des indicateurs d'état à plusieurs dates et d'évolution entre les dates permettront d'observer et de prendre la mesure de la rétraction spatiale du système de soins. La diminution de la présence des soins dans les villes, mesurée en termes absolus ou relatifs permettra de quantifier et localiser la rétraction.

Je postule que la fermeture des lits, des services et des établissements, des cabinets médicaux affecte préférentiellement les petites villes. C'est pourquoi je choisis de conduire cette recherche sur l'ensemble des 2233 unités urbaines ${ }^{15}$ de plus de 2000 habitants.

Je propose de détailler les phénomènes de concentration et de rétraction spatiale des services de soins pour différentes catégories de services : médecine de spécialité et pratique généraliste, établissements privés et publics. En effet, je fais l'hypothèse que ces catégories

\footnotetext{
${ }^{14}$ Barlet et Collin (2010) ont mobilisé les indices de Gini et de Theil pour mesurer la concentration de différentes catégories de services de soins (omnipraticiens, infirmiers, masseurs-kinésithérapeutes, spécialistes, pharmacies). Elles ont constaté que les généralistes, en 2007, au niveau des bassins de vie sont très

${ }^{15}$ Dans un premier temps la définition morphologique sera privilégiée afin d'appréhender le phénomène à l'échelon interurbain. Le désavantage est que cette définition morphologique de la ville ne tient pas compte des phénomènes de périurbanisation qui participent à l'expansion des villes et au maintien de leur rôle comme centre de services.
} 
s'inscrivent dans des schémas et des contraintes qui introduiront une certaine variété dans les phénomènes de concentration et de rétraction. Des contraintes essentiellement financières pour les établissements privés, politiques et financières pour les établissements publics, des contraintes de centralité (accès à une clientèle suffisamment nombreuse et aux ressources que constituent les laboratoires et les hôpitaux) pour la médecine de spécialité.

Delas (2011) et Lucas-Gabrielli (2000) ont observé que les établissements privés (très nombreux dans les années 1970 et de faible taille) sont entrés précocement dans des logiques de recompositions. Le processus de concentration spatiale a été important et s'est fait au profit de grands établissements positionnés sur des actes en hospitalisation de jour. Les ressorts de la recomposition ont été à la fois la nécessité de répondre à une demande solvable, l'ajustement aux modalités d'autorisation d'exercice, puis d'encadrement des tarifs des actes ${ }^{16}$. Je fais l'hypothèse que, toutes choses égales par ailleurs quand à la dynamique démographique régionale, la concentration se fait au profit des villes moyennes et grandes et que le pendant de la concentration est un processus de rétraction rapide qui affecte de manière privilégiée les petites villes.

Dans le cas de la médecine libérale, c'est la façon dont a évolué le numerus clausus régional et la part des médecins spécialistes, la manière dont s'organisent dans l'espace l'agrégation des micro-décisions des médecins qui s'installent et de ceux qui prennent leur retraite qui vont organiser des schémas de rétraction et de concentration. Pour Alouis (2013), les médecins suivent le mouvement économique général et les critères professionnels d'installation des médecins tiennent aux possibilités d'association, à la proximité d'un hôpital, à la connaissance antérieure du lieu par les stages ou les remplacements. Elle y ajoute des critères personnels (le cadre vie, les perspectives d'études pour les enfants, les perspectives d'activité professionnelles des conjoints). Bourgueil et Mousquès (2007) signalent quant à eux le rôle du lieu de formation pour expliquer le choix du lieu d'installation. Les phénomènes de désertification médicale étant apparus récemment, je suppose que la rétraction de la médecine générale est tardive et qu'elle a commencé très récemment. Elle n'affectera que les très petites villes. En revanche, pour la médecine de spécialité dont l'implantation est largement liée à la présence des établissements hospitaliers il est possible d'envisager qu'elle démarre plus précocement, et qu'elle affecte particulièrement la gynécologie-obstétrique, fortement dépendante de la fermeture des nombreuses maternités privées (1/4 des établissements privés en 1973) dans les années 1970 et 1980.

Dans le cas des établissements publics, le processus va être plus tardif et plus désordonné. En effet, pour les acteurs publics (l'Etat, les $\mathrm{ARS}^{17}$, les élus locaux, les directeurs d'établissements) il s'agit de résoudre une contradiction entre égalité d'accès aux soins et efficacité (sanitaire et financière) des structures, avec des positionnement qui pourront être

\footnotetext{
16 Tarification à l'Acte dite T2A

${ }^{17}$ Agence Régionale d'Hospitalisation (des organes décentralisés de l'Etat) qui ont géré les recompositions hospitalières. A partir de 2009 (loi HPST) les Agences Régionales de Santé (organes décentralisés de l'Etat) négocient les recompositions hospitalières (privé et public) et la continuité des soins en ambulatoire (médecine libérale). Désormais elles sont aussi chargées de mettre en place le parcours de soins des patients qui doit permettre un suivi coordonné entre les établissements et les médecins libéraux.
} 
variés selon qu'il s'agit de l'ARS, de l'Etat central, du maire ou du directeur de l'établissement de la municipalité concernée par une fermeture. Les négociations qu'impliquent cette multitude d'acteurs vont impacter les schémas de rétraction, de manière différenciée selon les besoins en soins locaux, selon l'importance des services de soins dans l'activité économique locale, selon l'équipement en soins de la région, selon les contraintes budgétaires de l'Etat. La logique de concentration défendue par des arguments de rentabilité et de sécurité se heurtera souvent à la volonté de maintenir une offre au plus prés des besoins (Fleuret, 1998).

Enfin, je souhaite aussi envisager l'organisation des phénomènes de rétraction et de concentration à l'échelle locale, en prenant appui sur le suivi de quelques métropoles à l'échelon de l'aire urbaine. L'hypothèse est qu'à cet échelon d'observation, on pourrait observer une concentration des établissements en périphérie des unités urbaines, une rétraction dans les centres des unités urbaines, et une apparition des praticiens autour des établissements en périphérie, dans les couronnes des pôles urbains.

\section{Existe-t-il une relation entre des phénomènes de rétraction et concentration des services de soins et les trajectoires démographiques des villes?}

Les travaux relatifs aux changements à l'œuvre dans le système urbain français signalent des processus de métropolisation (Paulus 2004) et de simplification de la trame urbaine (Bretagnolle, 2009) au point que Pumain et Paulus (2000) interrogent la possibilité d'une transformation durable de la structure du système urbain français. Cette transformation renforce les espaces métropolitains, points forts de la trame urbaine et affaiblit les petites villes en décroissance durable, souvent éloignées des espaces métropolitains. Les dynamiques démographiques des villes moyennes ou intermédiaires sont très influencées par celle de leur région: à l'ouest et au sud, les dynamiques sont favorables, au nord et à l'est leur croissance est souvent menacée.

Paulus (2004) repère 809 unités urbaines (la moitié des UU) situées dans l'espace à dominante rurale ou multi-polarisé, dont la croissance est 5 fois plus faible que la croissance moyenne de l'ensemble des villes (0,3\% par an, période 1954-1999). Ces petites villes décroissantes se trouvent dans toutes les régions même si les décroissances les plus fortes se rencontrent surtout dans les massifs montagneux et dans les vieilles régions industrielles ${ }^{18}$. Wolff et al (2013), dans une approche qui s'inscrit dans le courant des recherches sur les villes en rétraction ${ }^{19}$ mettent aussi en valeur des situations de déclin absolu entre 1975 et 2007 pour 69 des 354 aires urbaines françaises. Les $3 / 4$ de ces villes comptent moins de 50000 habitants, sont situées au cœur des vieux bassins miniers et industriels, dans le Nord, l'Est et sur les pourtours du massif central. Ces phénomènes de décroissance sont déjà apparus dans

\footnotetext{
${ }^{18}$ Dans ces dernières, des villes de grandes tailles comme Béthune, Lens, Douai, Valenciennes, Saint-Etienne apparaissent aussi en décroissance.

${ }^{19}$ Les villes en décroissance, en rétraction peuvent être définies comme « des espaces urbains qui ont connu des pertes de population, un retournement économique, un déclin de l'emploi et des problèmes sociaux, symptômes d'une crise structurelle » (Martinez-Fernandez et al., 2012)
} 
l'histoire des villes françaises (Pumain, 1982; Guérin-Pace, 1993), la nouveauté est le renforcement de cette autocorrélation temporelle au profit des grandes régions métropolitaines, au détriment des petites villes.

Bretagnolle (2009) interprète le déclin relatif des petites unités urbaines comme un effet de la contraction espace-temps qui court-circuite le rôle de desserte locale des petites villes tandis que Cunningham-Sabot et Fol (2010) y voient une incapacité de ces villes à valoriser leur situation et leurs potentialités dans le cadre de l'émergence d'une économie mondialisée très sélective.

Le deuxième axe de la recherche que je propose repose sur l'hypothèse que l'évolution de la répartition spatiale des soins pourrait accompagner ces transformations du système urbain : je postule l'existence de relations pour la période 1980-2010 entre la rétraction des services de soins et le déclin démographique des villes, entre la concentration des services de santé et la croissance des villes. Cette hypothèse s'appuie sur le caractère encore banal des services de soins, et sur le rôle de base économique qu'ils jouent dans certaines petites villes. Davezies (2012) revisite la théorie de la base économique et envisage que les activités de la fonction publique (santé, éducation, services de soins) soient considérées comme des activités de base pour les territoires qui les abritent. En m'inspirant de la formalisation du processus de décroissance décrit par Roth (2011), j'utiliserai deux scénarios pour illustrer mon propos.

Dans une ville de l'espace rural aux activités peu diversifiées, la décroissance démographique entraîne une diminution du nombre d'actes d'obstétrique conduits dans l'établissement hospitalier de la ville. L'Etat organise la fermeture du service de maternité ${ }^{20}$ et cette dernière entraîne le départ d'une partie des employés, la fermeture des services induits (restauration, blanchisserie, ambulance, commerces de vêtements et équipements pour nourrissons et petits enfants, commerces de proximité et/ou intermédiaire). Les médecins et soignants qui se sont installés dans les années 1960 ou 1970 arrivent à l'âge de la retraite. Ils cherchent des repreneurs mais le déclin démographique et économique de la ville la rend peu attractive et aucun repreneur ne se présente. La ville, après avoir perdu le service de maternité voit l'existence de l'hôpital menacé, perd ses soignants libéraux, son caractère répulsif se renforce: sa décroissance démographique se poursuit. Ainsi se dessine un scénario où décroissance et disparition progressive des services de soins se combinent et alimentent une boucle de rétroaction positive d'autant plus vive que la base économique de la ville est réduite.

A l'échelle du système des villes, la juxtaposition de tels scénarii dans un assez grand nombre de villes pourrait se traduire par la rétraction des services de soins sur l'ensemble du territoire. Cette rétraction concernerait en premier lieu les petites villes en déclin identifiées par Paulus (2004), Wolff et al (2013).

\footnotetext{
${ }^{20}$ Qui peut être envisagé comme une activité économique de base pour la ville.
} 
De la même manière, un scénario de concentration peut être dessiné. Il s'appuie sur les qualités des villes moyennes qui accueillent les Centres Hospitaliers. Ceux-ci sont dotés de plateaux techniques (bloc opératoire, laboratoire d'analyse, équipement d'imagerie médicale) dont le coût est relativement élevé et leur existence est conditionnée par la taille du bassin de population: quelques dizaines de milliers d'habitants au moins. La présence de tels équipements attire les jeunes médecins spécialistes libéraux souhaitant s'installer d'autant qu'elle s'accompagne de la possibilité de travailler à l'hôpital et en cabinet ${ }^{21}$ (Tonnelier et Vigneron, 1999 ; Salem et al., 2000 ; Barbat-Bussière, 2008 ; Coldefy et al., 2011). Cette attraction professionnelle est renforcée par le fait que la base économique de la ville présente une certaine diversité et offre des opportunités personnelles (emploi pour le conjoint, écoles secondaires voire enseignement supérieur pour les enfants). L'attraction exercée sur les jeunes médecins peut aussi renforcer la base économique médicale de la ville: la recherche appliquée en lien avec l'hôpital s'y développe. La fermeture d'un service de chirurgie ou d'une maternité dans une petite ville voisine a pu contribuer à renforcer l'activité médicale de la ville moyenne : Lerouvillois et Vinclet (2002) signalent par exemple les effets positifs de la fermeture des services de chirurgie et d'obstétrique à St-Hilaire du Harcouët au profit d'Avranches. Enfin, l'amélioration de l'accessibilité de cette ville (par la réalisation d'infrastructures routières par exemple) renforce son attractivité pour les soins : BarbatBussière (2009) signale ainsi que l'amélioration de l'accessibilité de Clermont-Ferrand a contribué à atrophier l'aire d'influence des établissements des petites villes d'Ambert, d'Issoire ou de Thiers. Depuis 2000, l'intégration de Vichy dans l'aire de Clermont a diminué l'offre des établissements privés vichyssois. Les travaux de Paulus (2004) sur les trajectoires relatives des villes entre 1968 et 1990 (eu égard à la composition de leur population active) étayent aussi cette hypothèse : en effet, Paulus observe l'émergence d'une catégorie de villes dans lesquelles les fonctions de services à la population environnante comme les services de santé, l'éducation et l'action sociale semblent s'être substituées en partie aux fonctions commerciales traditionnellement considérées comme centrales.

Ainsi, à l'échelle du système urbain dans son ensemble, rétraction et concentration pourraient se combiner pour renforcer d'un côté les spécialisations, l'attractivité, de l'autre le déclin, la perte de base économique.

\section{Envisager la rétraction comme un processus de diffusion spatiale}

La diffusion spatiale concerne des processus qui mettent en jeu des déplacements de matière, de produits, de personnes, de pratiques ou d'idées dans un ensemble (Saint-Julien, 1983). La

\footnotetext{
${ }^{21}$ Salem et al. (2000) observent que la présence des cardiologues, gynécologues-obstétriciens, pédiatres est beaucoup plus concentrée que celle des généralistes et monte à $95 \%$ dans les villes de plus de 20000 habitants (2000). Les travaux de Coldefy et al. (2011) soulignent aussi la répartition plus inégalitaire des médecins spécialistes : plus la spécialité est rare, plus les spécialistes sont concentrés : ainsi les ophtalmologistes sont présents dans 3,3\% des communes et sont directement accessibles à 50\% de la population tandis que les urologues sont présents dans $0,8 \%$ des communes, directement accessibles à $25 \%$ de la population. Les mêmes auteurs identifient les seuils de taille des unités urbaines à partir desquels on compte un praticien de spécialité : de 5000 habitants pour les ophtalmologues il passe à 10000 pour les urologues. Or depuis le milieu des années 1990, le nombre de médecins spécialistes formés a cru plus rapidement que le nombre de généralistes.
} 
réduction, voire la disparition des services de soins, établissements ou cabinets de ville pourrait-elle être interprétée comme un tel déplacement dans l'espace des villes ? La théorie de la diffusion spatiale a été essentiellement mobilisée pour formaliser la propagation des innovations et des épidémies dans l'espace (Hägerstrand, 1952 ; Pedersen, 1970 ; Pred, 1975 ; Gould, 1983 ; Saint-Julien, 1983 ; Rogers, 2003). Coldefy (2010) a observé la diffusion des établissements de santé mentale dans le réseau urbain français entre la fin du XIXe et la fin du XXe siècle.

Dans le cas de la réduction de la présence des services de soins dans le système urbain, ceuxci ne constituent ni une innovation, ni une épidémie. Si l'absence de services de soins se généralise à un nombre suffisant de villes elle pourrait cependant être envisagée comme le déplacement de l'absence. Grosjean (2005) dans un travail empirique sur la diffusion des clubs de football en Franche-Comté a observé une phase de rétraction, postérieure aux 4 phases de diffusion. Pendant cette phase, les clubs de foot des communes les moins peuplées de Franche-Comté ont fermé. Cette rétraction s'explique par le déclin de la demande de club de foot dans ces espaces. Dans le registre des soins, Lefebvre (2015) a noté une phase de concentration d'un réseau de cliniques franchisées en Inde. Cette phase de concentration succède à un processus de diffusion des franchises dans le réseau urbain. Elle s'explique par la mise en œuvre par la direction de la firme d'une stratégie de contrôle plus poussé des cliniques et d'évaluation plus sévère des propositions d'ouverture de cliniques par les franchisés.

Je propose de développer ces observations et de m'inspirer des principes des processus de diffusion spatiale énoncés par Hägerstrand (1952), Saint-Julien (1983) pour envisager la rétraction comme un processus de disparition des services de soins d'une partie des espaces dans lesquels ils sont présents au début des années 1980.

Tout processus de diffusion prend place dans un système fait d'éléments susceptibles d'adopter l'innovation. Ici les villes sont susceptibles d'enregistrer les fermetures, d'adopter la disparition des services de soins, et de participer au processus de rétraction.

Les processus de diffusion comportent un enchaînement chronologique en 4 phases et je fais l'hypothèse qu'il en va de même dans le cas de la rétraction. Pendant la phase d'apparition, dite aussi primaire, les villes qui «adoptent» l'innovation, c'est à dire la fermeture de services ou établissements de soins sont peu nombreuses, le processus de rétraction est lent. La phase de propagation qui vient ensuite correspond à une croissance forte du nombre de villes dans lesquelles se produisent les disparitions : la vitesse de propagation des fermetures s'accroît (c'est la $2^{\mathrm{e}}$ phase dite de diffusion) jusqu'à un point d'inflexion où le nombre de villes adoptantes continue de croître mais plus lentement : c'est la $3^{\text {e }}$ phase dite de condensation. La phase de saturation qui apparaît en dernier correspond à une croissance proche de 0 du nombre de villes dans lesquelles se produisent les fermetures. L'hypothèse ici est donc que pour la rétraction comme pour la diffusion, les étapes de la rétraction sont ordonnées, lente d'abord puis rapide, et lente à nouveau une fois que l'ensemble des villes susceptibles d'enregistrer la fermeture d'établissements ou de services, ou de cabinets de 
soins a été atteint. L'identification des phases du processus à partir des vitesses de diffusion des fermetures sera confrontée aux phases de planification de la carte sanitaire, ainsi qu'à l'évolution du numerus clausus et de la démographie médicale. J'essayerai de tester l'idée qu'il existe des relations entre la rétraction des établissements de soins et celles des cabinets de soins de villes : l'une commence-t-elle avant l'autre ? sont-elles synchrones ? ont-elles les mêmes vitesses?

Dans la théorie de la diffusion spatiale des innovations, l'interaction spatiale organise la propagation de l'innovation dans les systèmes urbains en s'appuyant sur deux canaux principaux : le voisinage pour le fonctionnement local, et la hiérarchie pour le fonctionnement interurbain. Ainsi, l'innovation percole par le canal hiérarchique des villes les plus grandes vers les plus petites. Dans le cas de la rétraction, l'hypothèse est que le mécanisme s'organise à l'opposé : la disparition des soins progresse des petites villes (qui enregistrent préférentiellement le déclin), vers des villes plus grandes. Le canal du voisinage paraît possible aussi à mobiliser pour les phénomènes de rétraction et concentration à l'échelon local : fermeture d'une clinique au profit d'une autre dans la même ville, ou au profit d'une autre dans une ville du même territoire (Bichier et Fellinger, 2005).

Dans les processus de diffusion spatiale, des barrières se manifestent pour stopper ou ralentir la diffusion. Dans le cas de la rétraction, plusieurs types de barrières peuvent être envisagées. Un premier type de barrière tiendrait aux dynamiques démographiques de croissance des villes : si les villes sont en croissance, l'utilité économique des services de soins n'est pas remise en cause, stoppant la rétraction pour ces villes, mais n'excluant pas des phénomènes internes de concentration. Un autre type de barrière pourrait tenir à la capacité de mobilisation des acteurs locaux contre la disparition ou la fermeture des services de soins, ou à leur capacité à mobiliser des politiques favorables au maintien : Chevillard montre ainsi que la capacité des acteurs locaux à s'organiser est un facteur essentiel de la mise en place des Maisons Pluridisciplinaires de Santé et de la lutte contre les « déserts médicaux » (Chevillard, 2015).

La théorie de la diffusion spatiale des innovations contribue à expliquer les différences de croissance économique et démographique entre types de villes à un moment donné : un cycle d'innovation est temporairement favorable au développement d'un ensemble de villes. Il se diffuse ensuite à l'ensemble des villes favorisant ainsi leur croissance. A la fin du cycle, tout le système a été atteint par l'innovation et s'est translaté : la structure s'est maintenue. En sera-t-il de même pour la rétraction ? On peut d'ores et déjà supposer que la rétraction d'un service banal comme les soins ne peut atteindre tous les éléments du système : elle reste cantonnée à un ensemble de villes où son utilité économique est faible. La rétraction introduit alors une différence entre des villes affectées par la disparition des soins et les villes épargnées. La rétraction pourrait alors agir comme une forme de perturbation interne et introduire un changement dans la structure du système, en favorisant l'apparition de soussystèmes aux trajectoires différenciées : celui des villes qui arrivent à maintenir un rôle de place centrale et maintiennent ainsi leur croissance, celui des villes qui perdent ce rôle sans pour autant en acquérir de nouveau et entrent alors en décroissance. 


\section{Conclusion}

A travers cette proposition, je souhaite apporter des éléments au débat sur la répartition des activités médicales et sur l'accès aux soins dans une perspective dynamique et évolutive. Comment la concentration et la rétraction opèrent-elles selon différentes catégories de services de soins ? Au bénéfice de quelles villes et de quelles régions opèrent-elles ? $\mathrm{Au}$ détriment de quelles villes? Toutes choses égales par ailleurs, la concentration est-elle plus sensible du côté de la médecine libérale et des établissements privés (dans lesquels on peut supposer que la logique financière est plus prégnante) que dans les établissements publics qui doivent répondre à des enjeux d'équité dans l'accès aux soins et de modernisation en matière d'équipement? Un deuxième enjeu réside dans l'inscription de ces questions dans celle plus large de la dynamique du système urbain : comment le système de soins s'adapte-t-il aux changements qui marquent l'évolution de la répartition de la population sur le territoire français : la répartition des activités de soins s'adapte-t-elle à la dynamique démographique du système urbain pour favoriser les localisations dans les grandes villes au détriment du premier niveau du système urbain? Un troisième enjeu réside dans la possibilité d'identifier des processus de rétraction en leur transposant les cadres de la théorie de la diffusion spatiale des innovations.

* Je remercie chaleureusement Sylvie Fol, Thérèse Saint-Julien, Lena Sanders pour les remarques et conseils qu'elles m'ont apportés.

\section{Bibliographie}

Alouis K. (2013). La géographicité des médecins libéraux, application à la métropole parisienne de la fin du XIXe siècle à 2012. Thèse soutenue à l'Université de Paris X Nanterre, le 3 décembre 2013, Volume 1, 278 p.

Barbat-Bussière S. (2008). L'offre de soins en milieu rural, l'exemple d'une recherche appliquée en Auvergne. Presses Universitaires Blaise Pascal, CERAMAC Clermont-Ferrand, $488 \mathrm{p}$.

Barlet M., Collin C. (2010). Localisation des professionnels de santé libéraux. Rapport pour la DREES, $56 \mathrm{p}$.

Bichier E., Fellinger F. (2005). Les fermetures de chirurgies et maternités publiques en France, Bilan de 9 années (1997-2005) et prospective. Rapport pour la Conférence Nationale des Centres Hospitaliers, 14 p.

Bourgueil Y., Mousquès J., Tajahmadi A. (2007). Comment améliorer la répartition géographique des professionnels de santé ? Les enseignements de la littérature internationale et des mesures adoptées en France. Rapport IRDES n 1635.

Bretagnolle A. (2009). Villes et réseaux de transport: des interactions dans la longue durée. Dossier de candidature pour une Habilitation à Diriger des Recherches, Vol. 3. Géographie. Université Paris I, 205 p.

Charreire H., Combier E., Michaut F., et al. (2011). « Une géographie de l'offre de soins en restructuration : les territoires des maternités en Bourgogne ». Cahiers de géographie du Québec, Vol. 55 n¹56, pp. 491-509. 
Coldefy M. (2010). De l'Asile à la ville : une géographie de la prise en charge de la maladie mentale en France. Thèse de doctorat soutenue le 22 juin 2010, Université Paris 1 PanthéonSorbonne, $519 \mathrm{p}$.

Coldefy M., Com-Ruelle L., Lucas-Gabrielli V., Marcoux L. (2011). Les distances d'accès

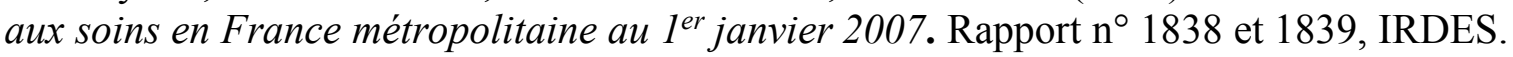

Chevillard G., Mousquès J., Lucas-Gabrielli V., Bourgueil Y., Rican S., Salem G. (2013). Maisons et pôles de santé : place et impact dans les dynamiques territoriales d'offre de soins en France, DT n ${ }^{\circ}$ 7, Novembre 2013, IRDES, 56 p.

Cunningham-Sabot E. et Fol S. (2010). " Déclin urbain et « Shrinking cities » : une approche critique de la décroissance urbaine ». Annales de géographie, 2010/4, n674, pp. 359-383.

Davezies L. (2012). La République et ses territoires, la circulation invisible des richesses, Col La république des idées. Paris : Seuil, $110 \mathrm{p}$.

Delas A. (2011). «L'hôpital public, un nouvel acteur territorial entre aménagement sanitaire et rivalités stratégiques ». Hérodote, 4/2011 (n 143), pp. 89-119.

Fleuret S., Séchet R.(dir) (2002). La santé, les soins, les territoires, Penser le bien-être. Rennes : PUR, $236 \mathrm{p}$.

Faure H., Lucas V., Tonnellier Fr. (1993). Offre et soins médicaux, évolution départementale 1968-1990. CREDES, Biblio n 992, Septembre 1993, 59 p.

Gould P. (1983). «Epidémiologie et maladie ». In Bailly A., Ferras R., Pumain D. (dir.), Encyclopédie de géographie. Paris : Economica, pp. 947-969.

Grosjean F. (2005). « La diffusion du football en Franche-Comté : la spatialisation du cycle de vie d'un service sportif ». Staps, 2005/2 n68, pp. 41-54.

Guérin-Pace Fr. (1993). Deux siècles de croissance urbaine, La population des villes françaises de 1831 à 1990. Paris : Anthropos, Coll. Villes, 205 p.

Hägerstrand T. (1953). Innovation diffusion as a spatial process, translation by Pred 1967. Chicago : University Press, 334 p.

IRDES (2015). Historique des réformes hospitalières en France, Mise à jour : Avril 2015, 36 p.

Jousseaume V. (2002). « Diffusion et recomposition de l'offre médicale : l'exemple des Pays de la Loire (1967-2000) ». In Fleuret S., Séchet R. (dir.), La santé, les soins, les territoires, Penser le bien-être. Rennes : PUR, pp. 33-49

Labasse J. (1980). L'hôpital et la ville, Géographie Hospitalière. Paris : Hermann Editeurs des Sciences et des Arts, $240 \mathrm{p}$.

Lalande Fr., Scotton C., Bocquet P.Y., Bonnet J.L. (2012). Fusions et regroupements hospitaliers : quel bilan pour les 15 dernières années. Rapport à l'Inspection Générale des Affaires Sociales, RM2012-020P, Mars 2012, 273 p.

Lefebvre B. (2015). "Emergence, diffusion et déclin d'un réseau de cliniques franchisées en Inde. Cybergeo : European Journal of Geography, Espace, Société, Territoire, document 710, mis en ligne le 19 février 2015, consulté le 08 octobre 2015. URL: http://cybergeo.revues.org/26756 ; DOI : 10.4000/cybergeo.26756

Lerouvillois G., Vinclet P. (2002). «Les hôpitaux sous contraintes : des restructurations aux recompositions de l'offre, l'exemple de la Basse-Normandie ». In Fleuret S., Séchet R. (dir.), La santé, les soins, les territoires, Penser le bien-être. Rennes : PUR, pp. 49-65.

Lucas-Gabrielli V, Tonnellier F., 2000, « Les équipements hospitaliers en France ». In Mattei M.F., Pumain D. (dir.), Données Urbaines 3. Paris : Anthropos, Col Villes, pp. 189-198.

Martinez-Fernandez C., Audirac I., Fol S., Cunningham-Sabot E. (2012). « Shrinking Cities : Urban Challenges of Globalization », International Journal of Urban and Regional Research, vol. 36, No.2, 213-225. URL : DOI : 10.1111/j.1468-2427.2011.01092.x.

Pedersen P. O. (1970). «Innovation Diffusion within and between National Urban Systems ». Geographical Analysis, 2: 203-254. doi: 10.1111/j.1538-4632.1970.tb00858.x 
Paulus F., Pumain D. (2000). « Trajectoires de villes dans le système urbain». In Mattei M.F., Pumain D. (dir.), Données Urbaines 3. Paris : Anthropos, Col Villes, pp. 189-198.

Paulus F. (2004). Coévolution dans les systèmes de villes: croissance et spécialisation des aires urbaines françaises de 1950 à 2000. Thèse de doctorat, Soutenue à l'Université Paris I Panthéon Sorbonne, le 14 Décembre 2004, 408 p.

Pumain D. (1982). La dynamique des villes. Paris : Economica, $230 \mathrm{p}$.

Pumain D., Saint-Julien Th. (2001). Les interactions spatiales. Paris : Armand Colin, col Cursus Géographie, 191 p.

Pred A. (1975). " Organizational spatial structure and city-system development », Economic Geography, Vol 51 n³, pp. 252-268.

Rogers E.M. (2003). Diffusion of innovations. New York : Free Press, 550 p.

Roth H. (2011). «Les « villes rétrécissantes » en Allemagne ». Géocarrefour, Vol 86/2, 2011.

Saint-Julien Th. (1983). «Diffusion spatiale». In Bailly A., Ferras R., Pumain D. (dir.), Encyclopédie de géographie. Paris : Economica, pp pp. 559-583.

Roth H. (2016). «Du déclin à la périphérisation : quand les courants constructivistes et critiques revisitent les différenciations spatiales en Allemagne ». Cybergeo: European Journal of Geography, Espace, Société, Territoire, document 758, mis en ligne le 4 janvier 2016, consulté le 07 janvier 2016. URL: http://cybergeo.revues.org/27389; DOI : $10.4000 /$ cybergeo. 27389

Schmitt B., Niel X. (1999). «La diffusion des services sociaux et sanitaires depuis 1980 ». Etudes et Résultats, Septembre 1999, n³2, DREES, 8 p.

Tonnellier Fr., Vigneron E. (1999). Géographie de la santé en France. Paris : PUF, Coll. Que sais-je ?, 127 p.

Vigneron E. (2001). Distance et santé, la question de la proximité des soins. Paris : PUF, coll. 'Médecine et société', $127 \mathrm{p}$.

Vigneron E. (dir.) (2002). Pour une approche territoriale de la santé. Datar, Editions de l'Aube, Coll. Bibliothèque des territoires, $286 \mathrm{p}$.

Vigneron E., Haas S. (2009 Janvier). 10 ans de recomposition hospitalière. Rapport pour la Direction des Etudes, Dexia Crédit Local, 16 p.

Wolff M., Fol S., Roth H., Cunningham-Sabot E. (2013). «Shrinking Cities, villes en décroissance : une mesure du phénomène en France ». Cybergeo : European Journal of Geography, Aménagement, Urbanisme, document 661, mis en ligne le 8 décembre 2013, consulté le 11 juin 2014. URL : http://cybergeo.revues.org/26136 ; DOI : $10.4000 /$ cybergeo. 26136 